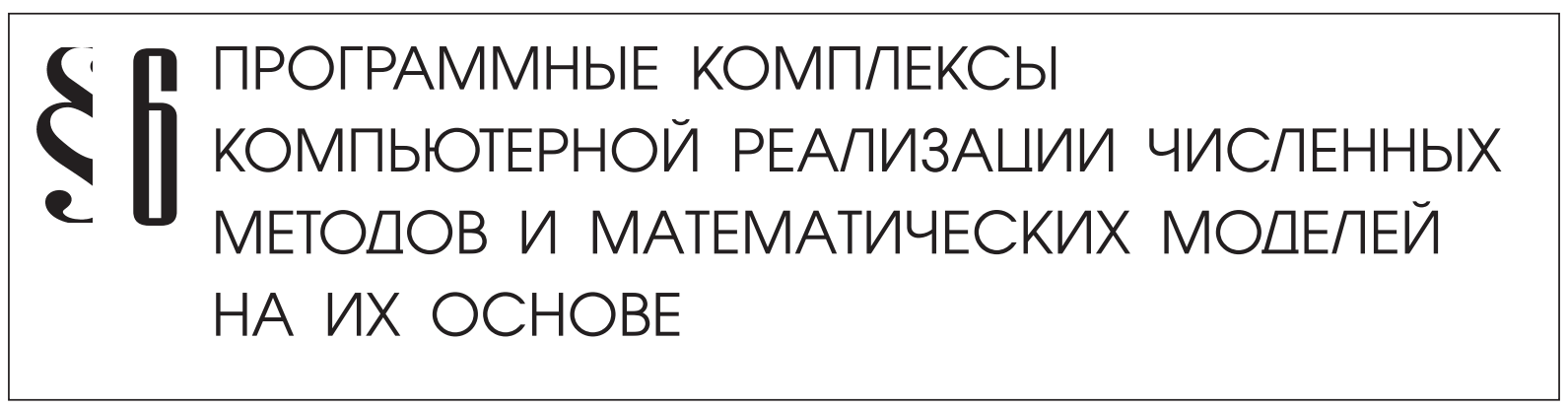

Олейникова С.А.

\title{
ЧИСЛЕННЫЕ МЕТОДЫ ОПТИМИЗАЦИИ ПЛАНИРОВАНИЯ СЛОЖНЫХ ПРОЕКТОВ ПРИ НАЛИЧИИ ВРЕМЕННЫХ И РЕСУРСНЫХ ОГРАНИЧЕНИЙ И ОБОБЩЕННОГО РЕСУРСНОГО КРИТЕРИЯ
}

Аннотация:Объектом исследования в данной работе являются сложные обслуживающие системы, отличительной особенностью которых является стохастический характер длительности обслуживания комплекса взаимно-зависимых работ, которые определяют проект. Целью работы является формирование план-графика по обслуживанию заявок с учетом ограничений на время обслуживания заявки и на объемы ресурсов, которыми располагает система. В качестве критерия оптимизации выбран обобщенный ресурсный критерий, позволяющий в зависимости от предпочтений пользователя описать такие режимы работы, как равномерная загрузка системы, максимальная загрузка и т.д. Методы построения план-графика основываются на численных алгоритмах, в основе которых лежат эвристики, определенные в результате анализа существующих подходов и специфики выдвинутой чели исследования. В результате предложен комплекс численных методов, которые легли в основу программного средства, формирующего план-график по обслуживанию заявок. Новизна заключается в применении эвристик, позволяющих получить субоптимальное расписание за приемлемое время. В качестве одной из областей применения результатов представлена оптимизация функционирования вагоноремонтного производства

Ключевые слова: алгоритм, оптимизация, временные ограничения, ресурсный критерий, эвристики, численный метод, возврат, план-график, программное средство, вагоноремонтное производство

Abstract: The objects of the research are the complex service systems, the distinctive feature of which is the stochastic duration of the servicing of the complex of mutually dependent works that define the project. The aim of the work is to develop a schedule of service requests taking into account restrictions on the time of service and on the amount of resources. As an optimization criterion a generalized resource criterion is selected. This criterion allows describing such modes as uniform system load, 
maximum load, etc depending on the preferences of the user. Methods of building the schedule are based on the numerical algorithms, which are formed with the help of heuristics. This heuristics are determined by the analysis of the existing approaches and specific objectives of the study. As a result the study proposes a complex of numerical methods that constitute the basis of the software for building the schedule service applications. The novelty of the study consists in the application of heuristics that provide a suboptimal schedule in a reasonable time. As one of the applications of the results the article shows the optimization of functioning of the wagon repairing production.

Keywords:numerical method, heuristics, resource criteria, time constraints, optimization, algorithm, back-tracking, schedule, software, wagon repairing production

\section{Введение}

Эффективность функционирования сложных обслуживающих систем во многом определяется качеством составленного расписания. Наличие целого ряда жестких ограничений на ресурсы и на длительность выполнения проектов, представляющих собой совокупность взаимно-зависимых работ, существенно усложняет процесс его составления. В связи с этим разработка методов формирования план-графиков с точки зрения некоторого критерия является весьма актуальной и практически значимой задачей.

В настоящее время наиболее распространенным (и, как следствие, наиболее изученным) является критерий скорейшего завершения проекта. Однако, существует целый ряд систем, для которых время целесообразно использовать не как критерий, а как ограничение. В этом случае рациональным вариантом является использование некоторого обобщенного ресурсного критерия, который может позволить описать различные варианты состояния системы (например, равномерная загрузка, минимизация простоя, максимизация загрузки и т.д.). В связи с этим, задача именно с таким критерием выбрана для исследования.

В качестве результата предлагаются алгоритмы, позволяющие получить расписание, близкое к оптимальному, с точки зрения заданного критерия за приемлемое время.

Полученные методы могут быть использованы для оптимизации любых систем, особенностями которых является случайное время выполнения комплекса взаимно-зависимых работ, временные ограничения и произвольный ресурсный критерий. В работе рассмотрена специфика применения результатов для оптимизации работы ремонтных систем на примере вагоноремонтного производства.

\section{1. Постановка задачи и ее особенности}

Математическая постановка задачи была приведена и исследована в [1]. Пусть на вход обслуживающей системы поступает поток проектов, требующих выполнения множества $W$ последовательно-параллельных работ:

$$
W=\left\{w_{1}, \ldots, w_{n}\right\} .
$$


Каждая работа $w_{i} \in W$ проекта задается:

- длительностью обслуживания dlit $_{i}$;

- объемом ресурсов необходимых $r_{i}=\left(r_{i 1}, \ldots, r_{i m}\right)$, где $m$ - общее количество видов ресурсов (если ресурс некоторого типа $j$ не используется, то соответствующее значение $\left.r_{i j}=0\right)$;

- $\quad$ взаимной зависимостью (для задания взаимной зависимости для каждой работы $w_{i}$ достаточно определить множество $S_{i}=S\left(w_{i}\right)$ непосредственно предшествующих ей работ).

Таким образом:

$$
w_{i}=w_{i}\left(\text { dlit }_{i} ; r_{i} ; S_{i}\right)
$$

Пусть проект $P$ также задан длительностью своего выполнения $T_{\text {max }}$ т.е.:

$$
P=P\left(W, T_{\max }\right) .
$$

Это означает, что выполнение проекта должно длиться не больше, чем $T_{\max }$.

Предполагается, что обслуживающая система располагает некоторым объемом ресурсов $R=\left(R_{1}, \ldots, R_{m}\right)$, где $m$ - число видов ресурсов.

Необходимо каждой работе поставить в соответствие такое время ее начала, чтобы выполнялись временные и ресурсные ограничения. Для формализации ограничений введем следующее множество. Пусть $W_{t}$ представляет множество работ, которые выполняются в момент $t$. Математически это множество можно определить как совокупность работ, которые начались раньше, чем $t$ (или непосредственно в этот момент), но время их завершения позже момента $t$ :

$$
W_{t}=\left\{w_{i} \in W: w_{i} \cdot t_{\text {нач }} \leq t<w_{i} \cdot t_{\text {нач }}+w_{i} \cdot d l i t\right\} .
$$

Тогда ограничения на длительность проекта и объем используемых ресурсов можно записать следующим образом:

$$
\left\{\begin{array}{l}
T_{\text {пр }} \leq T_{\max } \\
\sum_{j: w_{j} \in W_{t}} w_{j} . . r_{i} \leq R_{i}, \mathrm{i}=1, \ldots, \mathrm{m} .
\end{array}\right.
$$

Здесь $T_{\text {пр }}$ - время, затраченное на выполнение проекта $P$.

В качестве критерия оптимизации выберем произвольный ресурсный критерий. Это означает, что данный критерий так или иначе будет зависеть от объема ресурсов. Выбор данного критерия обусловлен следующими причинами [2]. Во-первых, существует достаточно большое количество проектов, для которых нет необходимости в скорейшем выполнении работ. Более того, расписание, составленное с точки зрения скорейшего критерия, будет неблагоприятно влиять на качество обслуживания (или другие пара- 
метры системы). В качестве примера таких проектов можно рассмотреть, например, множество дисциплин, подлежащих обучению студентами в заданный семестр, когда расписание стараются сформировать максимально равномерно в течение всего периода. Во-вторых, использование ресурсного критерия в совокупности с результатами планирования времени поступления тех или иных заявок может обеспечить сбалансированную работу системы с точки зрения ресурсов (эффективное использование оборудования и специалистов). В-третьих, для некоторых систем целесообразно обеспечить некоторый резерв производительности в те или иные моменты времени, когда велика вероятность появления внеплановых задач, требующих незамедлительного решения. Все вышеперечисленные особенности можно учесть, сформировав эффективный ресурсный критерий и временные ограничения. Следует отметить универсальность данного критерия. В частности, описав данную критериальную функцию с помощью константы, можно достичь равномерной загрузки системы во времени. Если значение такой константы будет совпадать с общим объемом имеющихся ресурсов, то получим критерий максимизации загрузки системы и т.д.

Введем в рассмотрение также функцию $f(t)=\left(f 1(t), \ldots, f_{m}(t)\right)$, определяющую наиболее целесообразный объем ресурсов, используемых в момент времени $t$. Исходя из этого, опишем критериальную функцию следующим образом. Пусть требуется минимизировать квадратическое отклонение фактического объема ресурсов, полученного при решении задачи планирования, от заданного в данный момент времени объема:

$$
\sum_{t} \sum_{i=1}^{m}\left(\sum_{j: w_{j} \in W_{t}} w_{j} \cdot r_{i}+R_{\text {исп і }}-f_{i}(t)\right)^{2} \rightarrow \min .
$$

Здесь $R_{\text {исп } i}$ - объем ресурсов, которые уже используются на этапе $i$ для выполнения других проектов.

Рассматриваемая задача относится к классу задач управления проектами. В целом данные задачи можно считать достаточно изученными. В частности, существуют подходы, позволяющие получить решения для задач скорейшего завершения проекта или максимизации прибыли [3-6]. Принципиальное отличие рассматриваемой задачи от изученных и решенных ранее заключается в наличии временных ограничений. Именно эта особенность в совокупности с многостадийностью существенно усложняет решение задачи, поскольку выполнение или нарушение ограничений можно проверить, как правило, лишь на финальных стадиях планирования, когда уже большинству работ назначено время их исполнения. В случае невыполнения условия необходимо формировать расписание заново, что существенно увеличивает время, затрачиваемое на решение задачи.

Таким образом, поиск подходов, позволяющих сформировать план-график по выполнению работ в описанных выше условиях за приемлемое время, является практически значимой задачей. 


\section{2. Обобщенный алгоритм формирования план-графика}

Разработаем алгоритм для исследуемой задачи с учетом ее особенностей. Очевидно, что в качестве основы необходимо будет использовать алгоритмы с возвратом, причем необходимо, по-возможности, минимизировать число возвратов [7]. С учетом NP-полноты задачи, необходимо разработать эвристики, которые позволили бы найти решение, близкое к оптимальному за приемлемое время [7].

Приведем первую, достаточно очевидную эвристику [8].

Эвристика 1. Пусть на данном этапе решается выбор о назначении работы $j$. Пусть общий объем используемых ресурсов в данный момент $t$ определяется вектором $R_{\text {исп }}(t)=\left(R_{\text {исп } 1}(t), \ldots, R_{\text {исп m }}(t)\right)$. Предположим, что имеют мест следующие неравенства:

$$
\left\{\begin{array}{l}
R_{\text {исп } 1}(t)+w_{j} \cdot r_{1} \leq f_{1}(t) ; \\
\cdots \\
R_{\text {исп } ~}(t)+w_{j} \cdot r_{m} \leq f_{m}(t) .
\end{array}\right.
$$

Они означают, что уровень используемых в данный момент ресурсов совместно с ресурсами, необходимыми для выполнения работы $j$, не достиг значения $f$. В этом случае работу необходимо выполнить в данный момент времени.

Предпосылки для использования данной эвристики основаны на том, что выполнение работы в данный момент времени, во-первых, улучшит значение частичной критериальной функции, отвечающей за этот этап, а, во-вторых, минимизирует нарушение временных ограничений, которые могут возникнуть из-за ее сдвига на более поздние периоды.

С помощью этой эвристики уже можно привести обобщенный алгоритм решения задачи. Предположим, что каким-либо образом (с помощью других эвристик) удалось выполнить планирование работ на произвольном временном этапе $i$ с точки зрения оптимальности целевой функции (5). Тогда обобщенный алгоритм будет иметь вид, представленный на рисунке 1.

Приведем пояснения к данному алгоритму. На предварительном этапе планирования осуществляется поиск временных характеристик работ (раннего и позднего времени, а также резерва) без учета ограничений на ресурсы и длительность.

Далее в цикле до тех пор, пока всем работам не будет назначено время начала, определяется множество работ $W_{i}$, которые можно выполнить в данное время, и вычисляется общий объем ресурсов, необходимый для их выполнения. После этого проверяются условия первой эвристики: если они выполняются, всем работам из $W_{i}$ назначается данное время. Однако, как правило, эти условия все же нарушаются. В этом случае необходимо определить, какие работы из $W_{i}$ будут выполнены в данное время, а какие перенесены на следующий этап. После этого оценивается новое время выполнения проекта: если оно превышает критическое значение, значит необходимо запретить существующее и искать новое решение. В противном случае осуществляется переход к следующему временному интервалу. 


\section{3. Детализация алгоритма}

Необходимо детализировать следующие этапы алгоритма:

- $\quad$ формирование план-графика работ на заданном временном интервале;

- оценка времени выполнения проекта;

- $\quad$ определение оптимального этапа для возврата

Поскольку вторая задача была уже рассмотрена в [9], а третья - в [10], остановимся подробно на планировании работ в данный момент времени. Для этого разработаем эвристики, позволяющие определить, какие из работ множества $W$ будут выполнены в данное время, а какие необходимо перенести на следующий временной интервал. Эвристики для одного частного случая решения данной задачи были разработаны в [8]. Теперь определим их для исследуемой задачи. Во-первых, будем пользоваться правилами, используемыми в алгоритмах при скорейшем завершении работ, заключающиеся в приоритизации работ [3]. Они предлагают последовательно выбирать для планирования работы с наивысшим приоритетом (это работы с минимальным временным резервом). Очевидно, что все работы до выполнения ограничений (6) необходимо выполнять на данном этапе. Вопрос возникает о времени выполнения работы, требующей ресурсов, нарушающих ограничения (6), но не нарушающих ограничения на ресурсы (4). Для этого можно воспользоваться следующей эвристикой.

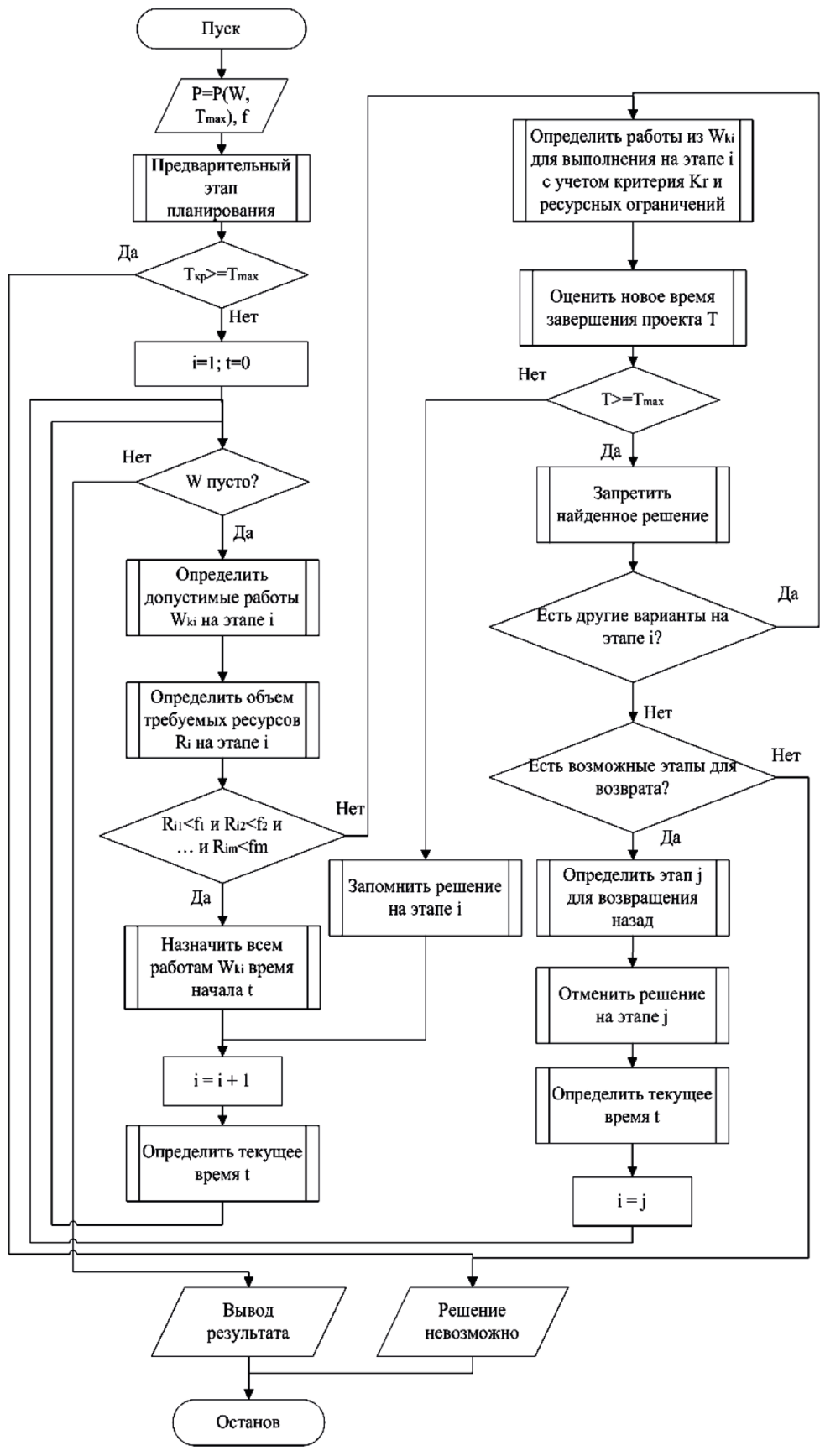

Рисунок 1 - 0бобщенный алгоритм решения задачи распределения работ по этапам 
Эвристика 2. Пусть на данном этапе нарушено хотя бы одно из неравенств (6). Работу необходимо выполнить в данное время в том случае, если будет справедливо неравенство:

$$
\begin{aligned}
& \sum_{i=1}^{m}\left(R_{\text {испі }}\left(j^{+}\right)-f_{i}(t)\right)^{2}+\sum_{i=1}^{m}\left(V\left(j^{-}\right)-\sum_{t>t_{0}}\left(f_{i}(t)-R_{\text {испі }}^{-}(t)\right)\right)^{2}< \\
& <\sum_{i=1}^{m}\left(R_{\text {испі }}\left(j^{-}\right)-f_{i}(t)\right)^{2}+\sum_{i=1}^{m}\left(V\left(j^{+}\right)-\sum_{t>t_{0}}\left(f_{i}(t)-R_{\text {испі }}^{+}(t)\right)\right)^{2} .
\end{aligned}
$$

Здесь:

- $\quad R_{\text {испі }}\left(j^{+}\right)$- объем используемых ресурсов типа $i$ с учетом выполнения работы $j$ на данном этапе;

- $\quad R_{\text {исп }}(j)$ - объем используемых ресурсов типа $i$ без учета выполнения работы $j$ на данном этапе;

- $\quad V_{i}(j)$ - общий объем ресурсов типа $i$, необходимый для выполнения всех незапланированных работ на всех оставшихся этапах при условии, что работа $j$ будет выполнена позже;

- $\quad V_{i}\left(j^{+}\right)$- общий объем ресурсов типа $i$, необходимый для выполнения всех незапланированных работ на всех оставшихся этапах при условии, что работа $j$ будет выполнена в данный момент.

Приведем формулы для расчета значений $V_{i}\left(j^{-}\right)$и $V_{i}\left(j^{+}\right)$.

$$
V_{i}\left(j^{-}\right)=\sum_{k \in W_{\text {nes }}}\left(w_{k} . . r_{i} \cdot w_{k} \cdot d l i t\right) .
$$

$W_{\text {нез }}$ - множество незапланированных работ (без учета работы $j$ ).

Формула для $V_{i}\left(j^{+}\right)$аналогичная и отличается только составом множества $W_{\text {нез' }}$ которое в данном случае будет содержать и работу $j$. Введем обозначения:

$$
\begin{aligned}
& K\left(t_{0}, j^{+}\right)=\sum_{i=1}^{m}\left(R_{\text {исп і }}\left(j^{+}\right)-f_{i}(t)\right)^{2}+\sum_{i=1}^{m}\left(V\left(j^{-}\right)-\sum_{t>t_{0}}\left(f_{i}(t)-R_{\text {испі }}^{-}(t)\right)\right)^{2} . \\
& K\left(t_{0}, j^{-}\right)=\sum_{i=1}^{m}\left(R_{\text {испі }}\left(j^{-}\right)-f_{i}(t)\right)^{2}+\sum_{i=1}^{m}\left(V\left(j^{+}\right)-\sum_{t>t_{0}}\left(f_{i}(t)-R_{\text {исті }}^{+}(t)\right)\right)^{2} .
\end{aligned}
$$

Тогда более компактно неравенство (7) можно переписать следующим образом:

$$
K\left(t_{0}, j^{+}\right)<K\left(t_{0}, j^{-}\right) .
$$

Поясним данную эвристику. Первое слагаемое представляет собой сумму, каждое слагаемое которой позволяет оценить степень близости текущего объема ресурсов данного типа к соответствующей целевой функции. Последующие - степень близости величины используемых ресурсов каждого типа, необходимой для выполнения всех 
незапланированных работ, к желаемому значению. В связи с этим, сумма этих двух слагаемых позволит получить примерную оценку целевой функции в случае, если спорные работы выполнять в данный момент времени или перенести на более поздние сроки.

Таким образом, алгоритм назначения работам времени в некоторым момент времени $i$ представлен на рисунке 2.

Как видно из рисунка 1, временные ограничения обуславливают необходимость использования процедуры возврата (back-tracking) при формировании план-графика. Следующая эвристика позволяет определить этап $l$ для возврата. Для этого введем специальный вектор $d o p_{l}=\left(\operatorname{dop}_{l 1}, \ldots, d o p_{l m}\right)$, каждый элемент которого показывает дополнительный резерв для выполнения еще одной работы $j$ на данном временном интервале. Данный вектор будет отрицательным в тех случаях, когда возвращение к данному интервалу нецелесообразно:

1. все работы, которые можно было запланировать запланированы;

2. при добавлении очередной работы нарушается
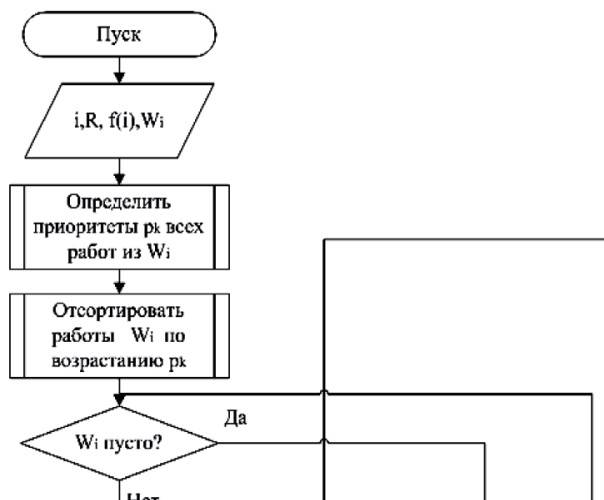

Назначить работе $j$

земя началта $t:$

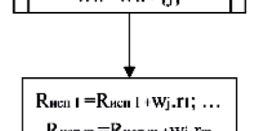

$\checkmark$ HeT

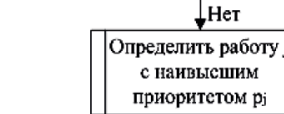

приорит сто

$\frac{1}{t}$

, R

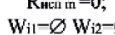

Heт
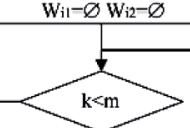
да

HeT
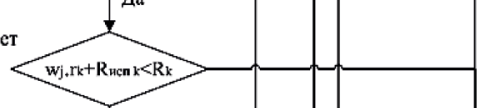

$\rightarrow r$ 1 Да $k-k+1$
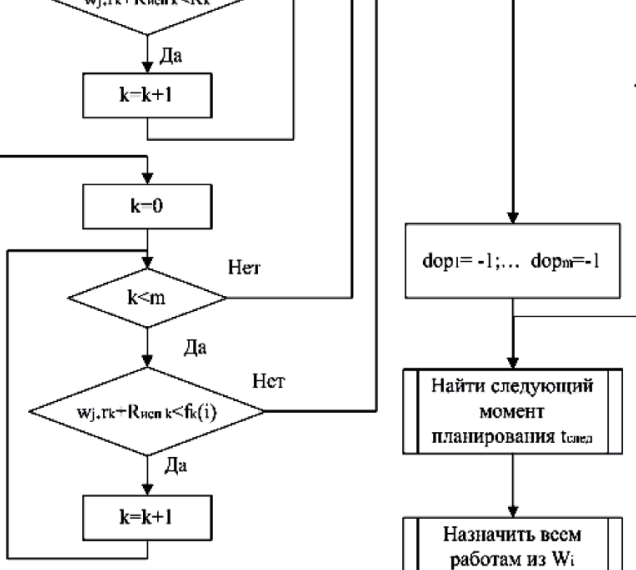

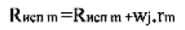

удалить работу ј из

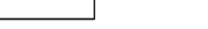

на і-м временном интервале одно из ограничений (6).

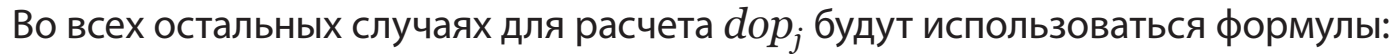

$$
d o p_{l k}=R_{k}-R_{k_{-} u c n}-w_{j} \cdot r .
$$


Эвристика 3. Пусть на некотором шаге было обнаружено нарушение временных ограничений (4). Тогда в качестве возврата целесообразно выбрать такой этап ј, для которого $d o p_{\text {јбудет }}$ являться решением задачи:

$$
\left\{\begin{array}{l}
\underset{1 \leq k \leq m}{\min d o p_{l k}} \rightarrow \max \\
d o p_{l k} \geq 0
\end{array} .\right.
$$

Условие (13) означает, что необходимо выбрать такой интервал, у которого минимальный резерв по всем видам ресурсов является наибольшим. В случае, если таких интервалов несколько, целесообразно выбрать более ранний интервал.

Для завершения описания обобщенного алгоритма необходимо рассмотреть подход к оценке времени завершения проекта. Пусть после планирования на некотором временном интервале определены объемы оставшихся работ $V=\left(V_{1}, \ldots V_{m}\right)$ аналогично формулам (9) и (10). Тогда минимальное время, за которое можно выполнить оставшиеся работы, определяется по формуле:

$$
T_{\min }=\max _{1 \leq l \leq m} \frac{V_{l}}{R_{l}} .
$$

В связи с этим, предварительную оценку времени можно выполнить следующим образом:

$$
T_{\text {ocm }}=T_{\text {mex }}+T_{\min }
$$

Таким образом, были разработаны численные методы, основанные на эвристиках, позволяющие решить оптимизационную задачу формирования план-графика при соблюдении временных и ресурсных ограничений и произвольного ресурсного критерия.

\section{4. Реализация алгоритмов и области применения полученных результатов}

Полученные алгоритмы могут использоваться для формирования план-графиков любых систем, особенностью которых является необходимость выполнения комплекса последовательно-параллельных работ со случайной длительностью, а также некоторый ресурсный критерий и временные ограничения. В качестве одной из областей применения полученных результатов является их использование в различных ремонтных предприятиях. Рассмотрим использование алгоритмов для формирования план-графиков по ремонту вагонов. Подробная постановка данной задачи была представлена в [11], а детальная разработка соответствующей программной системы - в [12]. Для хранения данных требуется база данных, содержащая сведения о вагонах, типах ремонта, выполняемых операциях, оборудовании, специалистах и т.д. Фрагмент структуры такой базы представлен на рисунке 3. 


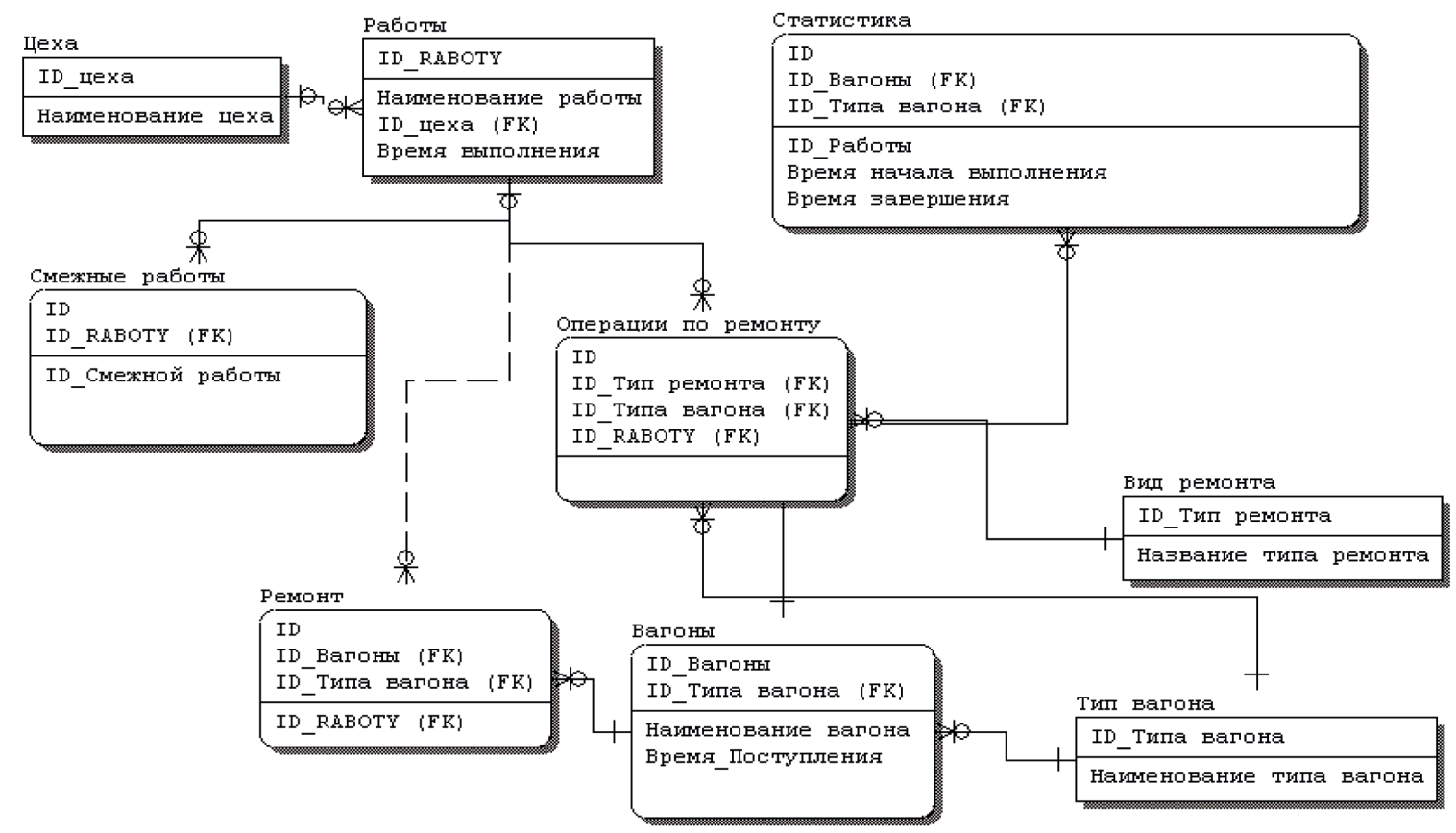

Рисунок 3 - Фрагмент структуры базы данных

Опишем основные этапы работы приложения. При поступлении вагона осуществляется его регистрация (рисунок 4).

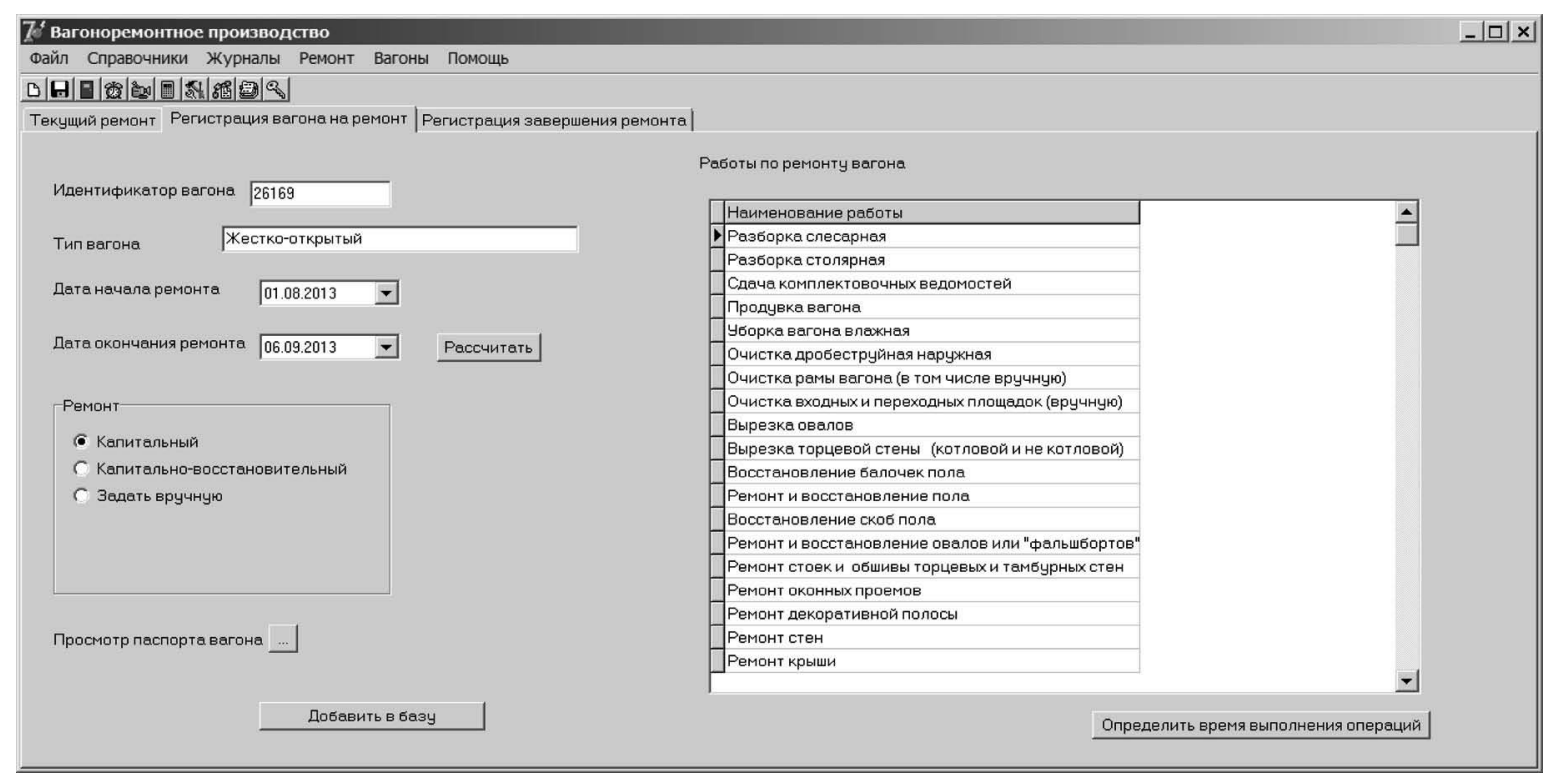

Рисунок 4 - Регистрация вагона на ремонт

Ее цель - найти все неисправности и определить перечень работ, необходимых для ремонта. Если выбрать определенный тип ремонта (например, капитальный или капитально-восстановительный), то перечень операций для ремонта будет сформирован автоматически. 
После регистрации необходимо выбрать команду меню «Ремонт/ Сформировать планграфик». При этом запустятся алгоритмы, представленные на рисунках 1 и 2. Результаты планирования будут отображены в специальном диалоговом окне (рисунок 5).

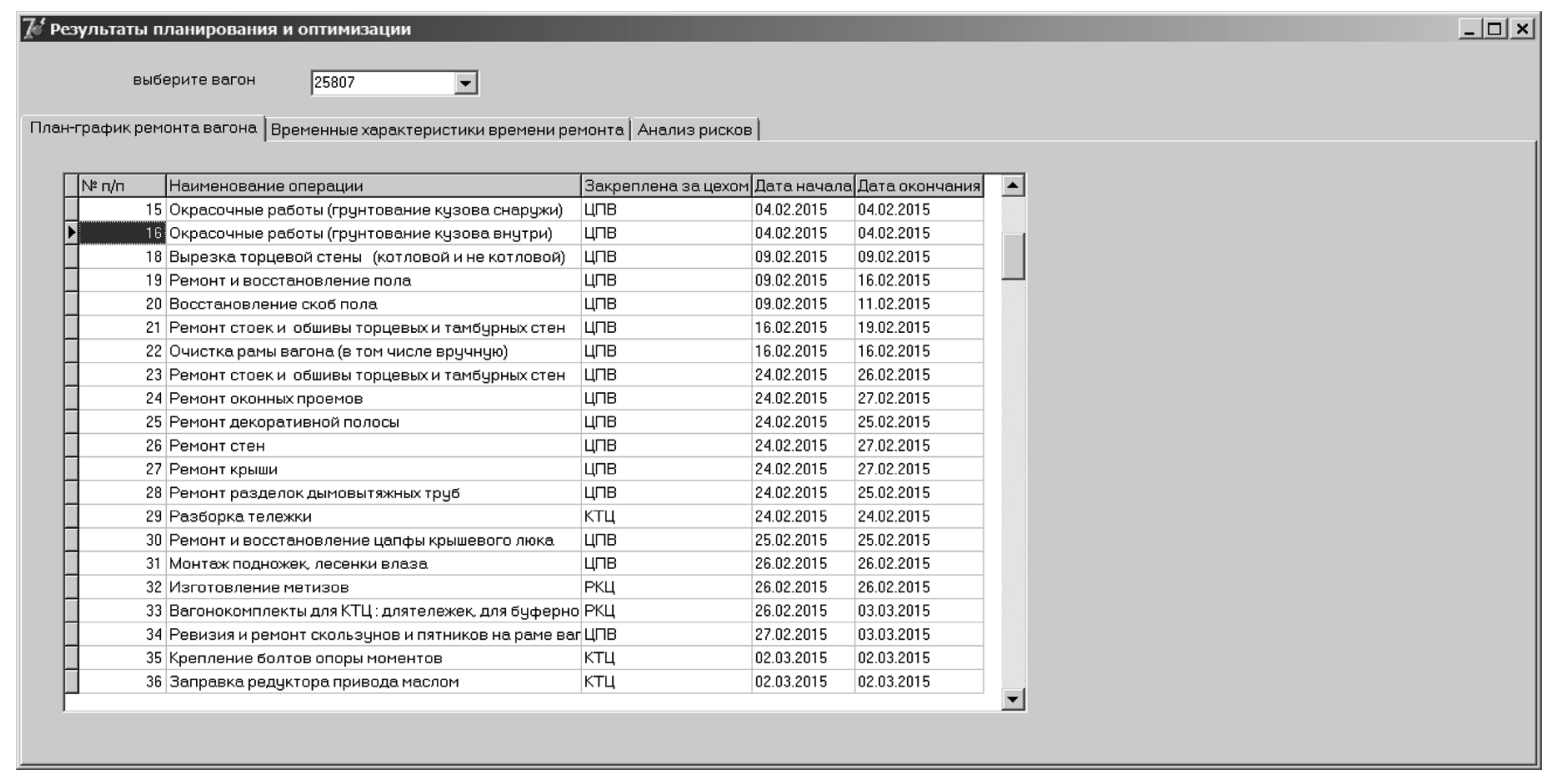

Рисунок 5 - Результаты планирования

Завершение каждой операции фиксируется, и время ее завершения заносится в базу данных. Это позволяет в любой момент времени отследить множество выполненных операций и множество операций, которые осталось выполнить для завершения ремонта вагона. Просмотреть соответствующую информацию можно на вкладке «Текущий ремонт» основного окна приложения.

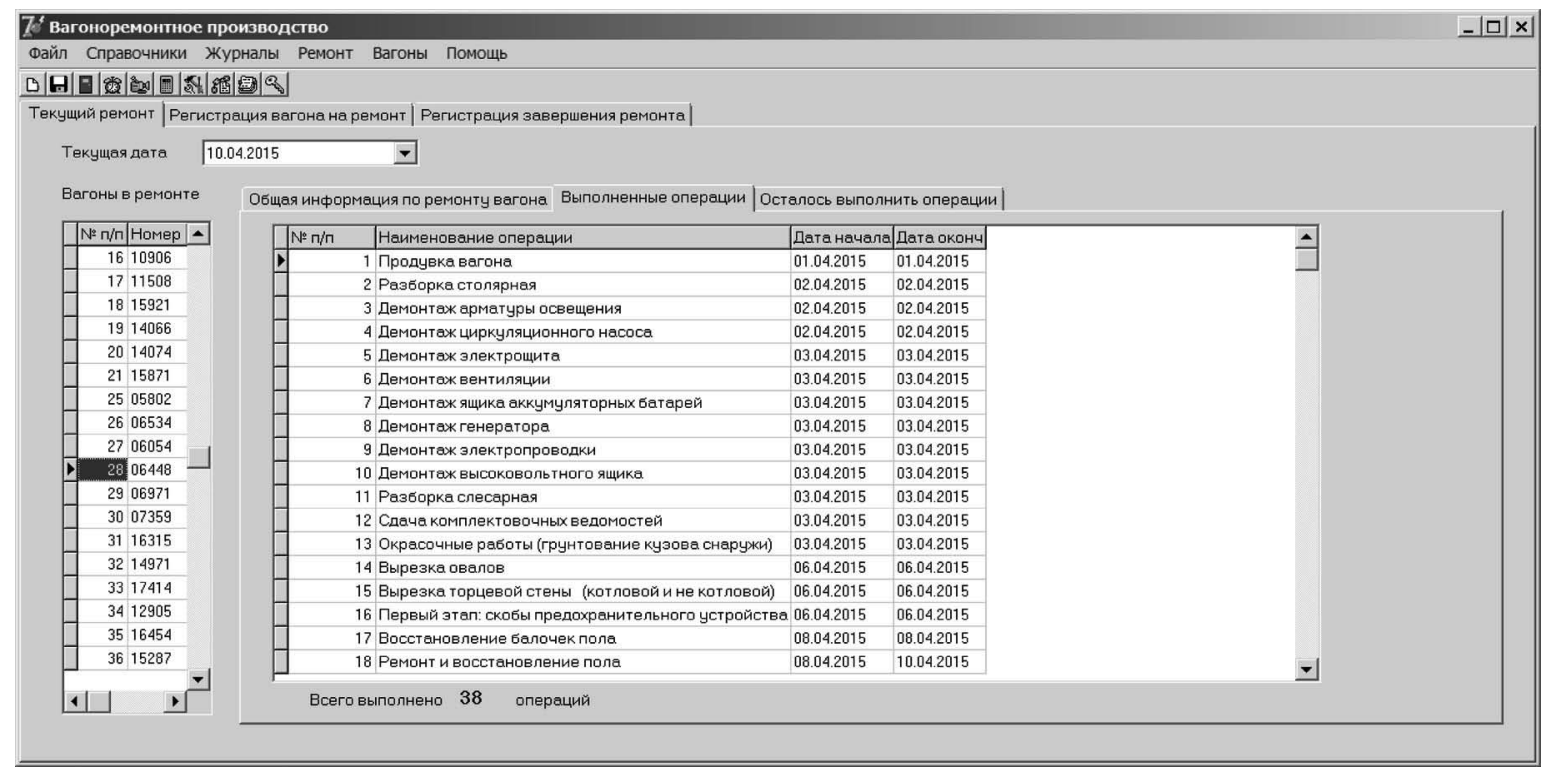

Рисунок 6 - Выполненные операции и операции, подлежащие выполнению 
Таким образом, реализовано приложение, позволяющее формировать план-график для ремонтных производств на примере вагоноремонтного завода, в основу которого положены разработанные эвристические алгоритмы планирования.

\section{Выводы}

В работе получены следующие результаты:

1. эвристики, позволяющие определить наиболее целесообразное время выполнения работ с точки зрения обобщенного ресурсного критерия, а также необходимость возврата из-за нарушения временных ограничений и этап для возврата;

2. численные методы, предназначенные для формирования план-графика проекта, учитывающие ресурсные и временные ограничения и обеспечивающие решение оптимизационной задачи с точки зрения произвольного ресурсного критерия;

3. программный комплекс, используемый для оптимизации функционирования сложных систем (на примере вагоноремонтного производства), путем автоматизации формирования расписания с использованием разработанных методов.

\section{Библиография :}

1. Олейникова С. А. Математическая модель и оптимизационная задача составления расписания для мультипроектной системы с временными и ресурсными ограничениями и критерием равномерной загрузки // Вестник Воронежского государственного технического университета.-2013.-Т9.-№ 6-3.-С. 58-61.

2. Олейникова С.А. Сравнительный анализ задач планирования работ с критериями скорейшего завершения проекта и равномерной загрузки системы // Системы управления и информационные технологии. - 2010.№ 4 (42).-C. 44-48.

3. Ахьюджа Х. Сетевые методы управления в проектировании и производстве. Пер. с англ. /Под. ред. В. Н. Калашникова. М.: Наука, 1979. - 640 с.

4. Голенко Д.И. Статистические методы сетевого планирования и управления. М.: Наука, 1968. - 400с.

5. Зуховицкий С.И., Радчик И.А. Математические методы сетевого планирования. М.: Наука, 1965. - 296с.

6. Кофман А., Дебазей Г. Сетевые методы планирования и их применение. М.: Прогресс, 1968. - 182с.

7. Кормен, Томас Х., Лейзерсон, Чарльз И., Ривест, Рональд Л., Штайн, Клиффорд. Алгоритмы: построение и анализ, 2-е издание. : Пер. с англ. - М. : издательский дом «Вильямс», 2005. - 1296 с.

8. Олейникова С.А. Разработка эвристик для принятия решения о времени выполнения работ для одной задачи управления проектами // Перспективы развития информационных технологий: сборник материалов IV Международной научно-практической конференции.-Новосибирск: Издательство НГТУ, 2011.-С. 44-49.

9. Олейникова С.А. Оценка критического времени в задачах управления проектами // Вестник Воронежского государственного технического университета. - 2011.-Т.7.-№ 2.-С. 106-109. 
10. Oleynikova S.A. Algorithm for determing the point of return for scheduling tasks with time constraints and criterion of uniform load// Modern informatization Problems: Proceedings of the XIX-th International Open Science Conference - Yelm, Wa, USA. - 2014.-C. 67 - 70.

11. Костючек В.А., Олейникова С.А. Математическое моделирование процесса планирования работ для вагоноремонтного производства // Информационные технологии моделирования и управления - 2008. № 6(49).-C.726- 730 .

12. Олейникова С.А. Разработка информационной системы для календарного планирования работ вагоноремонтного завода // Теоретические и прикладные вопросы современных информационных технологий. Материалы Х Всероссийской научно-технической конференции.-Улан-Удэ.-2009.-С.237-241.

13. Коробейников А.Г., Кутузов И.М., Колесников П.Ю. Анализ методов обфускации // Кибернетика и программирование. - 2012. - 1. - С. 31 - 37. URL: http://www.e-notabene.ru/kp/article_13858.html

14. Бородин А.В. Реконструкция и исследование датчика псевдослучайных чисел в VBA-подсистеме Microsoft Office // Кибернетика и программирование. - 2014. - 4. - С. 14 - 45. DOI: 10.7256/2306-4196.2014.4.12648. URL: http://www.e-notabene.ru/kp/article_12648.html

\section{References:}

1. Oleinikova S. A. Matematicheskaya model'i ioptimizatsionnayazadacha sostavleniya raspisaniya dlya mul'tiproektnoi sistemy s vremennymi i resursnymi ogranicheniyami i kriteriem ravnomernoi zagruzki // Vestnik Voronezhskogo gosudarstvennogo tekhnicheskogo universiteta.-2013.-T9.-№ 6-3.-C. 58-61.

2. Oleinikova S.A. Sravnitel'nyi analiz zadach planirovaniya rabot s kriteriyami skoreishego zaversheniya proekta i ravnomernoi zagruzki sistemy // Sistemy upravleniya i informatsionnye tekhnologii. - 2010.-№ 4 (42).-S. 44-48.

3. Akh'yudzha Kh. Cetevye metody upravleniya v proektirovanii i proizvodstve. Per. c angl. /Pod. red. V. N. Kalashnikova. M.: Nauka, 1979. -640 s.

4. Golenko D.I. Statisticheskie metody setevogo planirovaniya i upravleniya. M.: Nauka, 1968. - 400s.

5. Zukhovitskii S.I., Radchik I.A. Matematicheskie metody setevogo planirovaniya. M.: Nauka, 1965. - 2965.

6. Kofman A., Debazei G. Setevye metody planirovaniya i ikh primenenie. M.: Progress, 1968. - 182s.

7. Kormen, Tomas Kh., Leizerson, Charl'z l., Rivest, Ronal'd L., Shtain, Klifford. Algoritmy: postroenie i analiz, 2-e izdanie. : Per. s angl. - M. : izdatel'skii dom «Vil'yams», 2005. - 1296 s..

8. Oleinikova S.A. Razrabotka evristik dlya prinyatiya resheniya o vremeni vypolneniya rabot dlya odnoi zadachi upravleniya proektami // Perspektivy razvitiya informatsionnykh tekhnologii: sbornik materialov IV Mezhdunarodnoi nauchno-prakticheskoi konferentsii.-Novosibirsk: Izdatel'stvo NGTU, 2011.-S. 44-49.

9. Oleinikova S.A. Otsenka kriticheskogo vremeni v zadachakh upravleniya proektami //Vestnik Voronezhskogo gosudarstvennogo tekhnicheskogo universiteta. - 2011.-T.7.-№ 2.-S. 106-109.

10. Oleynikova S.A. Algorithm for determing the point of return for scheduling tasks with time constraints and criterion of uniform load// Modern informatization Problems: Proceedings of the XIX-th International Open Science Conference - Yelm, Wa, USA. - 2014.-S. 67 - 70. 
11. Kostyuchek V.A., Oleinikova S.A. Matematicheskoe modelirovanie protsessa planirovaniya rabot dlya vagonoremontnogo proizvodstva // Informatsionnye tekhnologii modelirovaniya i upravleniya - 2008. № 6(49).S.726- 730 .

12. Oleinikova S.A. Razrabotka informatsionnoi sistemy dlya kalendarnogo planirovaniya rabot vagonoremontnogo zavoda // Teoreticheskie i prikladnye voprosy sovremennykh informatsionnykh tekhnologii. Materialy X Vserossiiskoi nauchno-tekhnicheskoi konferentsii.-Ulan-Ude.-2009.-S.237-241.

13. Korobeinikov A.G., Kutuzov I.M., Kolesnikov P.Yu. Analiz metodov obfuskatsii // Kibernetika i programmirovanie. 2012. - 1. - C. 31 - 37. URL: http://www.e-notabene.ru/kp/article_13858.html

14. Borodin A.V. Rekonstruktsiya i issledovanie datchika psevdosluchainykh chisel v VBA-podsisteme Microsoft Office // Kibernetika i programmirovanie. - 2014. - 4. - C. 14 - 45. D0I: 10.7256/2306-4196.2014.4.12648. URL: http://www.e-notabene.ru/kp/article_12648.html 